http://dx.doi.org/10.32929/2446-8355.2018v27n4p385-395

\title{
ANÁLISE ESPACIAL DE POPULAÇÕES NATURAIS DE Dipteryx alata Vogel. SUBSÍDIO PARA COLETA DE SEMENTES
}

Diana Carla de Oliveira Santana Lima ${ }^{1}$, José Cambuim ${ }^{2}$, Alexandre Marques da Silva ${ }^{1}$, Marcela Aparecida de Moraes $^{2}$, Alan Rodrigo Panosso ${ }^{3}$, Mario Luiz Teixeira de Moraes ${ }^{4}$

\footnotetext{
${ }^{1}$ Mestre(a) no Departamento de Fitotecnia, Tecnologia de Alimentos e Sócio Economia, Universidade Estadual Paulista (UNESP), Faculdade de Engenharia de Ilha Solteira, Ilha Solteira - SP. *E-mail: dianac_lima@hotmail.com

${ }^{2}$ Doutor(a) no Departamento de Fitotecnia, Tecnologia de Alimentos e Sócio Economia, Universidade Estadual Paulista (UNESP), Faculdade de Engenharia de Ilha Solteira, Ilha Solteira - SP.

${ }^{3}$ Professor Adjunto do Departamento de Matemática, Universidade Estadual Paulista (UNESP), Faculdade de Engenharia de Ilha Solteira, Ilha Solteira - SP.

${ }^{4}$ Professor Adjunto do Departamento de Fitotecnia, Tecnologia de Alimentos e Sócio Economia, Universidade Estadual Paulista (UNESP), Faculdade de Engenharia de Ilha Solteira, Ilha Solteira - SP.
}

RESUMO: O padrão de distribuição espacial de espécies arbóreas é um dos fatores de relevante importância para o entendimento das relações ecológicas ocorridas em uma população ou comunidade florestal, possibilitando a compreensão de como determinada espécie utiliza dos recursos disponíveis para se estabelecer e reproduzir em determinado espaço. A interpretação dos resultados das análises espaciais de espécies arbóreas fornece subsídios para o entendimento desses fenômenos e indicação de indivíduos para programas de conservação genética e fomento florestal. Nesse contexto, o objetivo do trabalho foi subsidiar a coleta de sementes para conservação ex situ de seis populações naturais de baru (D. alata), com base na distribuição espacial, utilizando o Índice de Moran associado a um sistema de informação geográfica e estatística descritiva de seus caracteres silviculturais. As populações estão localizadas nos municípios de Paulo de Faria/SP, Campina Verde/MG, Ituiutaba/MG, Brasilândia/MS, Campo Grande/MS e Itarumã/GO. Todas as populações tiveram crescimento superior a $45 \mathrm{~cm}$ em relação a variável DAP (diâmetro à altura do peito). Levando em consideração que o DAP tem maior correlação dendrocronológica, a idade estimada dessas populações naturais estaria no intervalo de 40 a 63 anos. Com base nos valores de Índice de Moran, as populações de Ituiutaba e Itarumã, por apresentarem padrão de distribuição espacial aleatório, com alta variabilidade entre seus indivíduos, são preferencialmente indicadas para a coleta de sementes em programa de conservação ex situ da espécie.

Palavras-chave: Baru. Caracteres silviculturais. Conservação ex situ. Distribuição espacial. Índice de Moran. Sistemas de Informações Geográficas.

\section{SPATIAL ANALYSIS OF NATURAL POPULATIONS OF Dipteryx alata Vogel. GRANT FOR SEED COLLECTION}

ABSTRACT: The spatial distribution pattern of tree species is one of the factors of relevant importance for the understanding of the ecological relations that occur in a forest population 
or community, allow the understanding of how a species uses the available resources to establish and reproduce in a certain space. The interpretation of the results of the spatial analyzes of tree species provides grants for the understanding of these phenomena and indication of individuals for programs of genetic conservation and forest fostering. In this context, the objective of this work was to subsidize the collect of seeds for ex situ conservation of six natural populations of the Baru (D. alata), based on the spatial distribution, using the Moran Index associated to a geographic information system and descriptive statistics of their silvicultural characteristics. The populations are located in the municipalities of Paulo de Faria / SP, Campina Verde / MG, Ituiutaba / MG, Brasilândia / MS, Campo Grande / MS and Itarumã / GO. All the populations had growth superior to $45 \mathrm{~cm}$ in relation to the variable DAP (diameter at breast height). Taking into consideration that DAP has greater dendrochronological correlation, the estimated age of these natural populations would be in the range between 40 to 63 years. Based on the values of Moran Index, the populations of Ituiutaba and Itarumã, because present a pattern of random spatial distribution, with high variability among their individuals, are preferentially indicated for the collection of seeds in an ex situ conservation program of the species.

Key words: Baru. Silvicultural characters. Ex situ conservation. Spatial distribution. Moran index. Geographic Information Systems.

\section{INTRODUÇÃO}

A heterogeneidade espacial apresentada por diferentes sistemas naturais geralmente possui uma multiplicidade de fatores geradores, os quais têm recebido atenção especial de diversos ramos da ciência (DALE et al., 2002).

Espécies vegetais frequentemente apresentam distribuição espacial agrupada, isto é, formam grupos de indivíduos que possuem a mesma forma de reprodução, presença de distúrbios ou fatores ambientais que limitam sua distribuição, e até mesmo, pelas complexas interações entre membros da comunidade (BORCARD et al., 1992; PERRY; DIXON, 2002). Conhecer o padrão de distribuição das espécies e sua estruturação espacial são estratégias importantes para adotar medidas de manejo para preservação ou conservação da floresta. Estudos com essa abordagem vêm se tornando cada vez mais comuns, em virtude da disponibilidade de sistemas de informações geográficas (SIG). Esses sistemas permitem a visualização espacial de variáveis de interesse, como, por exemplo, índices de qualidade de vida ou distribuição das espécies por meio de mapas (DRUCK et al., 2004).

Diversos processos ecológicos estão relacionados à distribuição espacial de indivíduos de uma população em uma comunidade florestal. As síndromes de polinização e de dispersão de sementes, as relações intra e interespecífica. Como competição e a heterogeneidade edafoclimática, estabelecem a estrutura genética espacial de uma população (EPPERSON, 1992).

Os indivíduos em uma população podem apresentar três padrões básicos de distribuição espacial: aleatório, agrupado e regular (LUDWIG; REYNOLDS, 1988; KREBS, 1999). O padrão aleatório, em que a localização de um indivíduo não interfere na localização de outro 
da mesma espécie, se opõem ao padrão de distribuição agrupado (ou agregado), em que a presença de um recurso tem alta probabilidade de influenciar a localização dos indivíduos de uma mesma espécie, apresentando, assim, baixos índices de dispersão. No padrão de distribuição regular (disperso) a população apresenta alto índice de dispersão e os indivíduos possuem distâncias semelhantes uns dos outros (GREIG-SMITH, 1964; KERSHAW, 1973; MUELLER DOMBOIS; ELLENBERG, 1974; BARBOUR et al., 1987).

Apesar de existir a possibilidade de uma população apresentar distribuição regular, as plantas raramente se distribuem dessa maneira na natureza (BARBOUR et al., 1987). De acordo com Legendre e Fortin (1989), os seres vivos, em geral, tendem a se distribuir de forma agrupada devido ao ambiente ser estruturado espacialmente por várias formas de produção de energia, gerando assim processos irregulares de disponibilização de recursos. Dessa forma, os processos demográficos são influenciados pela heterogeneidade ambiental, gerando padrões espaciais que se distanciam da aleatoriedade e/ou regularidade (THOMAS; KUNIN, 1999).

A dispersão de sementes é o processo inicial que gera a distribuição espacial de uma espécie. Para uma espécie cujos diásporos são dispersos pelo vento (anemocoria), seria esperada uma distribuição dos indivíduos ao acaso, uma vez que não há a deposição proposital de diásporos em determinados micro-sítios, a não ser que fatores ambientais interfiram. Por outro lado, para a autocoria, sem auxílio de agentes externos, seria esperada a deposição de diásporos em determinados micro-sítios, mais precisamente ao redor da plantamãe. Tal padrão também seria esperado em espécies dispersas por animais (zoocoria), uma vez que muitas vezes há a deposição de diásporos em locais específicos. Caso fatores ambientais sejam mais importantes no estabelecimento das plântulas da espécie, padrões diferentes de distribuição espacial podem ser encontrados.

O Cerrado possui alta diversidade de plantas, entre as quais muitas apresentam possibilidades de exploração, cujo maior potencial é sua utilidade na alimentação. O baru (Dipteryx alata) faz parte do grupo de espécies nativas usadas pela população regional como fonte complementar de renda familiar, pela exploração extrativista do fruto. É uma das espécies frutíferas nativas mais promissoras para cultivo, em razão do seu uso múltiplo e da alta taxa de germinação de sementes e estabelecimento de mudas. É utilizada em reflorestamento de reservas legais e de proteção ambiental, margens de rios e córregos (SANO et al., 2004). Como a exploração do fruto se dá por extrativismo, e são ainda insuficientes as informações sobre a biologia e manejo do baru, é indispensável a realização de estudos que contribuam com o delineamento de estratégias mais eficientes para sua domesticação, conservação e uso sustentável (SILVA et al., 1997). A espécie também apresenta relevante importância ecológica e é classificada como espécie chave do Cerrado, pois seu fruto amadurece na seca e alimenta diversas espécies da fauna dessa região, inclusive o gado (SANO et al., 2004). Estudos, como os realizados por Oliveira (1998) e Rocha et al. (2002), têm mostrado variabilidade genética nos caracteres de crescimento (diâmetro e altura) em $D$. alata, indicando que esta espécie tem potencial de uso em programas de conservação e melhoramento genético. Assim, este estudo objetivou a coleta de sementes para conservação ex situ de seis populações naturais de D. alata, com base na distribuição espacial, utilizando o 
Índice de Moran associado a um sistema de informação geográfica e estatística descritiva de seus caracteres silviculturais.

\section{MATERIAL E MÉTODOS}

\section{Áreas de Estudo}

O estudo foi realizado em seis populações naturais de D. alata, localizadas nos municípios de Paulo de Faria/SP, Campina Verde/MG, Ituiutaba/MG, Brasilândia/MS, Campo Grande/MS e Itarumã/GO (Figura 1).

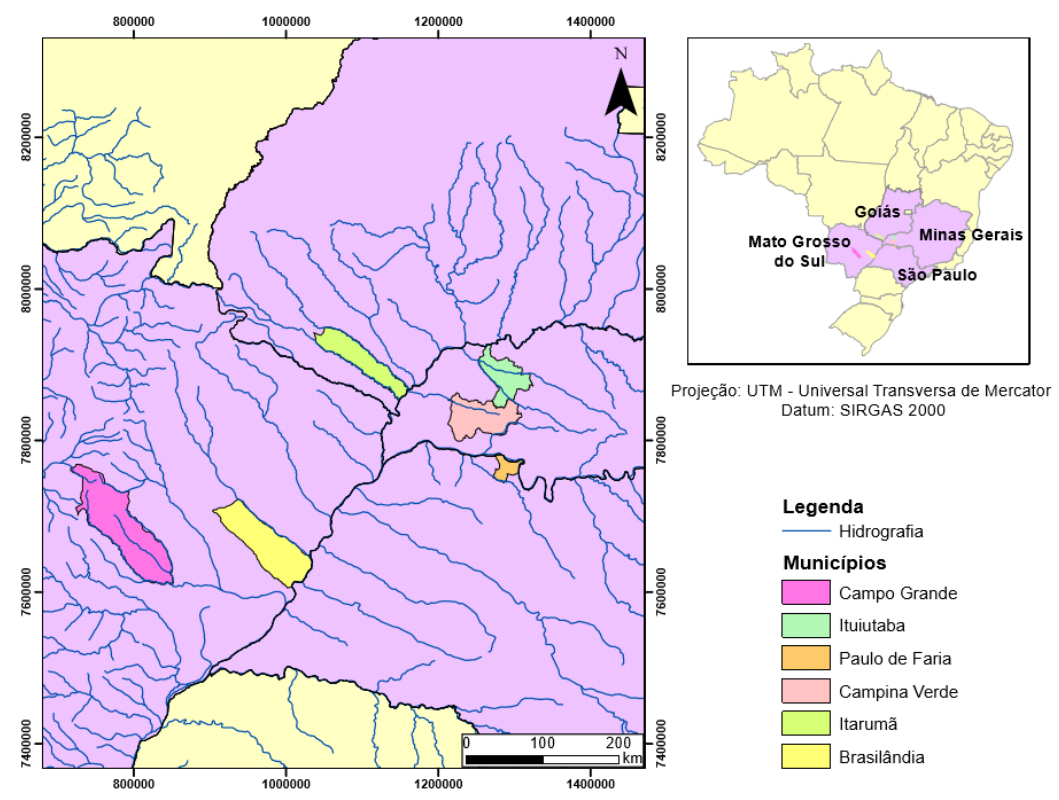

Figura 1. Localização das áreas de estudo. Location of study sites.

As características dos municípios em que se localizam as populações de $D$. alata evidenciam diferenças quanto à altitude, vegetação, clima, solo, relevo e pluviosidade, o que é interessante quando se busca a representatividade genética de uma espécie arbórea. Desse modo, a amostragem da população de Paulo de Faria (POP-PF) é composta por 61 árvores matrizes, que estão localizadas no entorno da Estação Ecológica de Paulo de Faria, em área de pastagens, canaviais e margens de rodovia. A população de Ituiutaba (POP-BA) é constituída por uma amostra de 42 árvores matrizes, que se localizam no interior de pastagens e à margem de rodovias. Também em áreas de pastagens encontra-se a amostra formada por 47 árvores matrizes da população de Campina Verde (POP-CV), que ocorrem com outras espécies arbóreas como aroeira (Myracrodruon urundeuva Allemão), pau-brasil (Caesalpinia echinata Lam. C) e jatobá (Hymenaea stigonocarpa Mart. ex Hayne). A população de Campo Grande (POP-CG) formada por 52 árvores matrizes localiza-se no entorno da área urbana- A população de Brasilândia (POP-BR) é representada por 38 árvores matrizes em uma área de pastagem contornada por plantações de eucalipto. A população de Itarumã (POP-MA), representada por 29árvores matrizes, localiza-se no interior de pastagens. Em todas as populações foram mensurados os caracteres silviculturais: altura total (ALT, m), diâmetro a altura do peito (DAP, $\mathrm{cm}$ ) e diâmetro médio da copa (DMC, $\mathrm{m}$ ) das árvores matrizes, e feito o seu georreferenciamento. 


\section{Estatística Descritiva}

As estimativas das estatísticas descritivas dos caracteres silviculturais foram obtidas utilizando-se o "modelo 105" do programa SELEGEN (RESENDE, 2007). Os parâmetros estimados foram: médias $(\hat{m})$, variância $\left(\hat{S}^{2}\right)$, desvio padrão $(\hat{S})$, coeficiente de variação ( $C V$ ), assimetria $\left(\hat{A}_{3}\right)$ e curtose $\left(\hat{A}_{4}\right)$, para cada um dos caracteres avaliados:

$$
\begin{aligned}
& \hat{m}=\frac{\sum x_{i}}{n} ; \quad \hat{S}^{2}=\frac{\sum_{i=1}^{n}\left(x_{i}-\hat{m}\right)^{2}}{n-1} \hat{s}=\sqrt{\hat{S}^{2}} C V=\frac{100 . \hat{s}}{\hat{m}} \\
& \hat{A}_{3}=\frac{\sum\left(x_{i}-\hat{m}\right)^{3}}{n \cdot \hat{s}^{3}} \hat{A}_{4}=\frac{\sum\left(x_{i}-\hat{m}\right)^{4}}{n \cdot \hat{s}^{4}}
\end{aligned}
$$

Estas estimativas foram descritas por Andriotti (2003).

\section{Autocorrelação espacial global}

A distribuição espacial dos fenótipos das árvores matrizes das seis populações naturais de D. alata, foi determinada a partir da estatística do Índice de Moran, pelo uso de seus caracteres quantitativos (ALT, DAP e DMC) e de suas coordenadas geográficas. As árvores matrizes foram georreferenciadas utilizando receptor GPS (Global Position System) da marca Garmin MAP 62sc.

A estimativa do Índice de Moran (I) foi obtida com base na expressão citada por Câmara et al. (2004):

$$
I^{(k)}=\frac{\sum_{i \neq j}^{n} w_{i j}\left(Z_{i}-\bar{Z}\right)\left(Z_{J}-\bar{Z}\right)}{\sum_{i=1}^{n}\left(Z_{i}-\bar{Z}\right)^{2}}
$$

em que: $n$ é o número de áreas, $Z_{i}$ é o valor do atributo considerado na área , $\bar{Z}$ é o valor médio do atributo na região de estudo, $w_{i j}$ são os elementos da matriz normalizada de proximidade espacial e $k$ é a ordem de vizinhança.

De forma geral, I varia entre $-1 \mathrm{a}+1$, não estando, no entanto, restrito a esse intervalo. No caso dos valores da variável de estudo serem semelhantes entre localizações vizinhas, verifica-se autocorrelação positiva (valor de I é positivo), caso contrário, a autocorrelação é negativa (valor de I é negativo). Se as observações estão distribuídas no espaço de forma aleatória não existe efeito espacial, tendendo a autocorrelação para um valor próximo de zero (FUCKS et al., 2004; WALLER; GOTWAY, 2004; O’SULLIVAN; UNWIN, 2010).

A técnica de autocorrelação espacial de Índice de Moran é uma estatística inferencial, ou seja, os seus resultados são sempre validados com base no teste de hipóteses que nos permite averiguar se o processo espacial que promoveu o padrão observado resulta de uma mudança aleatória (FUCKS et al., 2004).

Para o cálculo da autocorrelação espacial o software utilizado foi o ArcGIS ${ }^{\circledR}$ versão 10.2, que, após o cálculo do índice, determina um z-score e um p-value, para verificar a significância estatística dos dados. O p-value e o z-score são medidas de significância 
estatística que nos indicam a rejeição ou não da hipótese nula, ambos associados à distribuição normal, como representado na Figura 2.

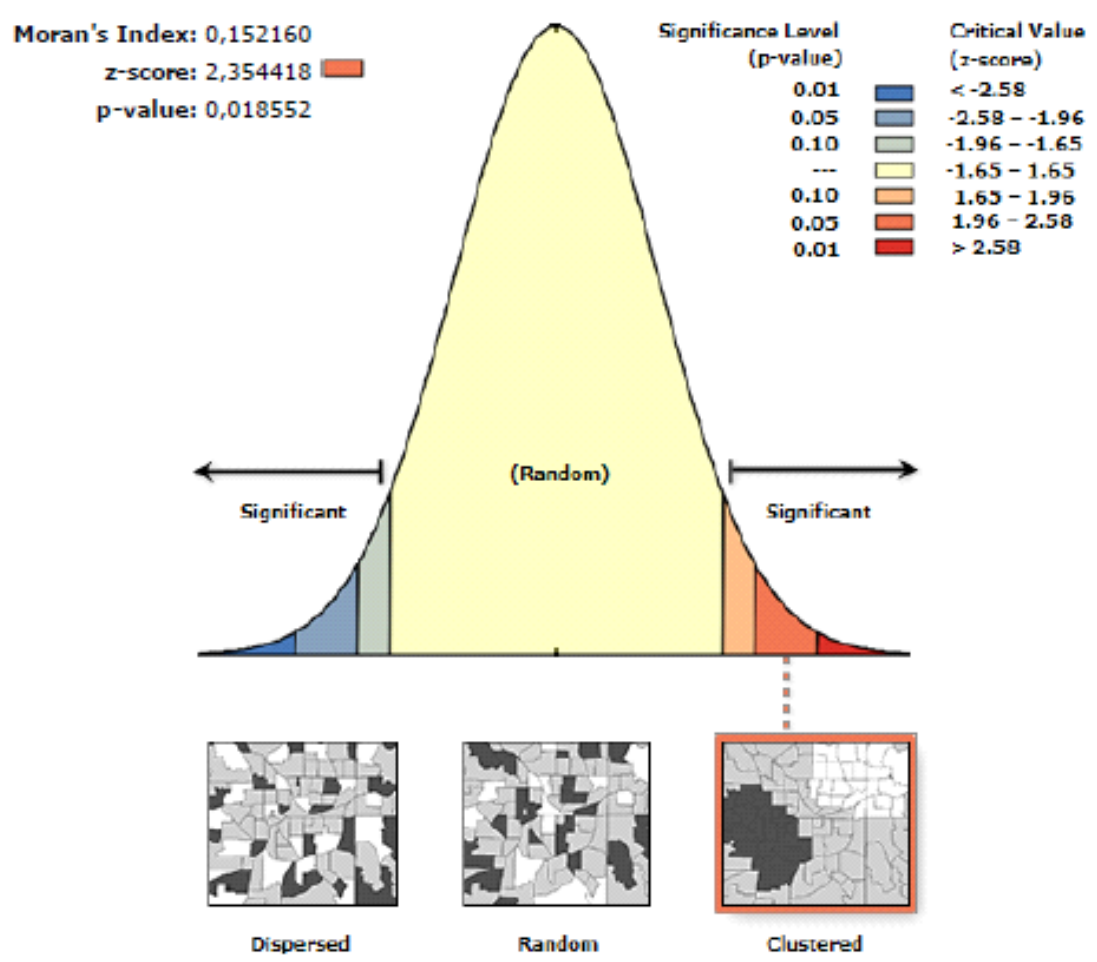

Figura 2. Exemplo de representação do resultado estatístico da Autocorrelação espacial global. Example of representation of the statistical result of the global spatial Autocorrelation.

\section{RESULTADOS E DISCUSSÃO}

\section{Estatística descritiva}

A média para o caráter altura variou de 14,63 m (POP-BA) a 17,82 m (POP-MA), correspondendo a uma média geral de 16,01 m (Tabela 1). Com base no coeficiente de variação, a população mais homogênea foi a POP-CV $(17,41 \%)$ e a mais heterogênea a POPBR $(22,71 \%)$. Em relação à distribuição dos dados, a maioria das populações apresentou uma distribuição do tipo simétrica e mesocúrtica. A única exceção foi a POP-PF, que apresentou assimetria negativa, mas quanto à curtose foi mesocúrtica.

No que se refere ao crescimento em DAP, todas as populações apresentaram crescimento superior a $45 \mathrm{~cm}$. Assim como no caráter altura, a POP-BA $(45,04 \mathrm{~cm})$ também apresentou menor incremento para o caráter DAP e a população com indivíduos que apresentaram maiores valores de DAP foi a POP-PF $(58,04 \mathrm{~cm})$ (Tabela 1). O IMA, para o DAP, obtido por Zaruma et al. (2015) foi de $0,91 \mathrm{~cm}$, valor semelhante $(1,12 \mathrm{~cm})$ ao obtido por Siqueira et al. (1992), sugerindo que, os indivíduos dessas populações tenham de 40 a 63 anos. Assim, no presente estudo, se for considerado as informações do caráter DAP ter maior correlação dendrocronológica a idade estimada dessas populações naturais estaria no intervalo entre 40 e 63 anos. A variação entre os indivíduos expressada pelo coeficiente de variação foi menor na POP-BR $(21,13 \%)$ e maior na POP-CG $(32,45 \%)$. Predominou nas populações a 
distribuição simétrica e mesocúrtica, com exceção da POP-BA que foi assimétrica positiva e leptocúrtica (Tabela 1). O IMA apresentado por Zaruma et al. (2015) foi de 0,36 m para o diâmetro médio de copa. Com base nesse caráter as populações poderiam ter de 36 (POP-PF) a 57 anos (POP-MA). A população com menor variação para esse caráter foi a POP-CV $(16,40 \%)$ e a maior a POP-MA $(48,30 \%)$. A POP-MA foi à única população que apresentou distribuição de dados do tipo assimétrica positiva e leptocúrtica.

Tabela 1. Estatística descritiva para os caracteres altura, diâmetro a altura do peito (DAP) e diâmetro médio da copa (DMC) para seis populações de Dipteryx alata (POP), localizadas nos municípios de Paulo de Faria (PF), Ituiutaba (BA), Itarumã (MA), Campo Grande (CG), Campina Verde (CV) e Brasilândia (BR). Descriptive statistics for height, diameter and breast height $(\mathrm{DBH})$ and mean diameter of the crown (MDC) for six populations of Dipteryx alata (POP), located in the municipalities of Paulo de Faria (PF), Ituiutaba (BA), Itaruma (MA), Campo Grande (CG), Campina Verde (CV) and Brasilândia (BR).

\begin{tabular}{|c|c|c|c|c|c|c|c|c|c|c|c|c|}
\hline \multirow{2}{*}{ POP } & \multicolumn{4}{|c|}{ ALTURA (m) } & \multicolumn{4}{|c|}{$\mathrm{DAP}(\mathrm{cm})$} & \multicolumn{4}{|c|}{$\operatorname{DMC}(\mathrm{m})$} \\
\hline & $\hat{m}$ & $C V$ & $\hat{A}_{3}$ & $\hat{A}_{4}$ & $\hat{m}$ & $C V$ & $\hat{A}_{3}$ & $\hat{A}_{4}$ & $\hat{m}$ & $C V$ & $\hat{A}_{3}$ & $\hat{A}_{4}$ \\
\hline $\mathrm{PF}$ & 15,97 & 21,11 & $\mathrm{AN}$ & $\bar{M}$ & 58,04 & 32,18 & $S$ & $\mathrm{M}$ & 13,06 & 23,50 & $S$ & $\mathrm{M}$ \\
\hline $\mathrm{BA}$ & 14,63 & 19,34 & S & M & 45,04 & 27,34 & AP & $\mathrm{L}$ & 13,07 & 18,91 & S & M \\
\hline MA & 17,82 & 18,10 & $S$ & M & 56,05 & 26,01 & S & M & 20,56 & 48,30 & AP & $\mathrm{L}$ \\
\hline $\mathrm{CG}$ & 16,09 & 21,28 & S & M & 57,38 & 32,45 & S & M & 13,31 & 21,86 & S & M \\
\hline $\mathrm{CV}$ & 16,08 & 17,41 & $S$ & M & 52,43 & 22,90 & S & M & 16,09 & 16,40 & S & M \\
\hline $\mathrm{BR}$ & 15,56 & 22,71 & $\mathrm{~S}$ & M & 46,78 & 21,13 & $\mathrm{~S}$ & M & 15,13 & 20,76 & $\mathrm{~S}$ & M \\
\hline
\end{tabular}

$\hat{m}$ Média geral; CV Coeficiente de variação (\%); $\hat{A}_{3}$ Assimetria; $\hat{A}_{4}$ : Curtose; AN: Assimétrica Negativa; S: Simétrica; AP: Assimétrica Positiva; M: Mesocúrtica; L: Leptocúrtica. $\hat{m}$ Average overall; $C V$ Coefficient of variation (\%); $\hat{A}_{3}$ Asymmetry; $\hat{A}_{4}$ : Kurtosis; AN: Negative Asymmetric; S: Symmetrical; AP: Positive Asymmetry; M: Mesokurtic; L: Leptokurtic.

\section{Índice de Moran Global}

A observação dos resultados de altura, DAP e DMC (Tabela 2) obtidos para as populações de Paulo de Faria e Brasilândia apontam que, para todos os caracteres silviculturais considerados, a distribuição espacial da espécie apresentou padrão agrupado, no qual os indivíduos tendem a estar localizados juntos, em ambas as populações. Estudos realizados por Rosseto et al. (2005) e Miranda (1992) com as espécies Xylopia aromatica e Caryocar brasiliense respectivamente, também nativas do Cerrado, foi observado padrão similar utilizando o mesmo coeficiente de autocorrelação espacial.

A análise dos valores de z e p indicaram que o I de Moran é estatisticamente significativo, e que existe probabilidade inferior a $10 \%$ de que este padrão agrupado possa ser resultado do acaso. Essa análise sugere que as árvores mais próximas têm maiores similaridades para os caracteres silviculturais analisados, e podem apresentar cruzamento entre indivíduos da mesma família. Sendo assim, a coleta de sementes dessas populações não seria prioritária para um programa de conservação $e x$ situ, em virtude da baixa variabilidade dos dados. Entretanto, a conservação desses materiais não deve ser descartada. 
Tabela 1. Autocorrelação espacial global para os caracteres altura, diâmetro a altura do peito (DAP) e diâmetro médio da copa (DMC) para seis populações de Dipteryx alata (POP), localizadas nos municípios de Paulo de Faria (PF), Ituiutaba (BA), Itarumã (MA), Campo Grande (CG), Campina Verde (CV) e Brasilândia (BR). Global spatial autocorrelation for height, diameter and breast height $(\mathrm{DBH})$ and mean diameter of the crown (MDC) for six populations of Dipteryx alata (POP), located in the municipalities of Paulo de Faria (PF), Ituiutaba (BA), Itarumã (MA), Campo Grande (CG), Campina Verde (CV) and Brasilândia (BR).

\begin{tabular}{llllll}
\hline POP & Caráter & $\begin{array}{l}\text { Moran's } \\
\text { Index }\end{array}$ & $z$-score & $p$-value & $\begin{array}{l}\text { Padrão } \\
\text { Espacial }\end{array}$ \\
\hline PF & ALT & 0,152 & 2,354 & 0,018 & Agrupado \\
PF & DAP & 0,104 & 1,693 & 0,090 & Agrupado \\
PF & DMC & 0,108 & 1,728 & 0,084 & Agrupado \\
\hline BA & ALT & 0,108 & 0,891 & 0,372 & Aleatório \\
BA & DAP & 0,135 & 1,150 & 0,250 & Aleatório \\
BA & DMC & 0,088 & 0,768 & 0,442 & Aleatório \\
\hline MA & ALT & 0,054 & 1,298 & 0,194 & Aleatório \\
MA & DAP & 0,008 & 0,652 & 0,514 & Aleatório \\
MA & DMC & 0,019 & 1,082 & 0,279 & Aleatório \\
\hline CG & ALT & $-1,128$ & $-3,488$ & 0,001 & Disperso \\
CG & DAP & $-0,216$ & $-0,627$ & 0,530 & Aleatório \\
CG & DMC & $-0,097$ & $-0,246$ & 0,806 & Aleatório \\
\hline CV & ALT & $-0,011$ & 0,222 & 0,824 & Aleatório \\
CV & DAP & 0,100 & 2,472 & 0,013 & Agrupado \\
CV & DMC & 0,013 & 0,709 & 0,478 & Agrupado \\
\hline BR & ALT & 0,127 & 1,915 & 0,056 & Agrupado \\
BR & DAP & 0,145 & 2,105 & 0,035 & Agrupado \\
BR & DMC & 0,111 & 1,704 & 0,088 & Agrupado \\
\hline
\end{tabular}

Para as populações de Ituiutaba e Itarumã, verificou-se que a distribuição espacial apresentou padrão aleatório (Tabela 2), no qual todos os indivíduos estão localizados independentemente uns dos outros. Dado os baixos valores obtidos para z $(0,89 ; 1,14$ e 0,76 , respectivamente para altura, DAP e DMC para a população de Ituiutaba; e 1,29; 0,65 e 1,08, respectivamente para a população de Itarumã), infere-se que o padrão não aparenta ser significativamente diferente do aleatório. Este resultado indica que nessas populações a coleta de sementes pode ser realizada aleatoriamente dentro destas amostras, em decorrência do alto grau de variabilidade observado entre os dados amostrais. Kageyama et al. (2003) também encontraram uma tendência ao padrão aleatório estudando uma população de Ocotea odorata. O mesmo padrão de distribuição foi verificado por Rosseto et al. (2005), ao estudarem uma amostra de população da espécie Vochysia tucanorum em um fragmento de Cerrado. Outros autores também não encontraram estruturação espacial para diversas espécies (a exemplo de Silva et al. (2005) com Caryocar brasiliense, Sebbenn et al. (1998) com Genipa americana, Souza et al. (2004) com Chorisia speciosa, e Aguiar et al. (2004) com Astronium fraxinifolium). 
A população de Campina Verde apresentou padrões de distribuição distintos entre os atributos altura e DMC e DAP (Tabela 2). Para a altura e o DMC, o padrão encontrado foi o aleatório, e os baixos valores de z (0,22 e 0,70, respectivamente) indicam que não há indícios de que o padrão encontrado seja diferente de aleatório. Por outro lado, o padrão de distribuição espacial encontrado para o atributo DAP foi o agrupado, com probabilidade inferior a 5\% de que este padrão agrupado tenha sido consequência do acaso.

Por fim, a população de Campo grande também apresentou padrões de distribuição diferentes entre os atributos DAP e DMC e altura (Tabela 2). Para o DAP e o DMC, o padrão encontrado foi o aleatório, e os baixos valores de z (-0,62 e -0,24, respectivamente) indicam que não há indícios de que o padrão encontrado seja diferente de aleatório. Por outro lado, o padrão de distribuição espacial encontrado para o atributo altura foi o regular, com probabilidade inferior a $1 \%$ de que este padrão de dispersão tenha sido consequência do acaso. No padrão regular, os indivíduos tendem a ser regularmente espaçados.

O estudo de autocorrelação espacial por meio do Índice de Moran se mostrou um método eficiente para esse tipo de análise, fornecendo informações significativas sobre os padrões espaciais. O uso de software de sistemas de informações geográficas também apresentou relevante utilidade para o cálculo da estrutura espacial das populações naturais. Assim, verificou-se que o padrão espacial de plantas é uma característica importante de comunidades ecológicas e a distribuição das populações vegetais discutidas nesse estudo representou um bom exemplo de como esses padrões podem diferir no contexto espacial. Pela análise integrada dos resultados da autocorrelação espacial global advinda do Índice de Moran e a da estatística descritiva, pode-se inferir que as populações apresentaram padrões espaciais aleatório, agrupado e regular.

\section{CONCLUSÕES}

Os indivíduos das populações de Dipteryx alata apresentaram, no geral, distribuição simétrica e mesocúrtica em relação aos caracteres estudados.

As populações de Ituiutaba e Itarumã exibiram padrão de distribuição espacial aleatório, apontando alta variabilidade entre seus indivíduos, desse modo a coleta de sementes para a conservação ex situ dessa espécie nessas amostras é preferencialmente indicada.

\section{REFERÊNCIAS BIBLIOGRÁFICAS}

AGUIAR, A. V.; COELHO, A. G. S.; MOURA, M. F.; MORAES, L. K.; PINHEIRO, J. B.; MORES, M. L. T.; ZUCCHI, M. I.; MOURA, N. F.; VENCOVSKY, R. Autocorrelação espacial de caracteres morfológicos em populações naturais de gonçalo-alves (Astronium fraxinifolium Schott). Bioscience of Journal, Pulau Pinang, v. 20, p.153-162, 2004.

ANDRIOTTI, J. L. S. Fundamentos de estatística e geoestatística. São Leopoldo: Editora Unisinos, 2003. 165 p.

BARBOUR, M. G.; BURK, J. H.; PITTS, W. D. Terrestrial Plant Ecology. 2. ed. Califórnia: Benjamim/Cummings, 1987. 634 p. 
BORCARD, D.; LEGENDRE, P.; DRAPEAU, P. Partialling out the spatial component of ecological variation. Ecology, Hoboken, v. 73, n. 3, p.1045-1055, 1992.

CÂMARA, G.; CARVALHO, M. S.; CRUZ, O. G.; CORREA, V. Análise espacial de áreas. In: DRUCK, S.; CARVALHO, M. S.; CÂMARA, G.; MONTEIRO, A. M. Análise espacial de dados geográficos. Planaltina, DF: Embrapa Cerrados, 2004. 208 p.

DALE, M. R. T.; DIXON, P.; FORTIN, M. J.; LEGENDRE, P.; MYERS, D. E.; ROSENBERG, M. S. Conceptual and mathematical relationships among methods for spatial analysis. Ecography, Malden, v. 25, n. 5, p.558-577, 2002.

DRUCK, S., CARVALHO, M. S., CÂMARA, G., MONTEIRO, A. V. M. Análise espacial de dados geográficos. Brasília, DF: EMBRAPA, 2004. 209 p.

EPPERSON, B. K. Spatial structure of genetic variation within populations of forest tree. New Forests, Dordrecht, v. 6, n. 1, p.257-278, 1992.

FUCKS, S.; CARVALHO, M. S.; CÂMARA, G.; MONTEIRO, A. M. V. Análise espacial de dados geográficos. Planaltina: Embrapa Cerrados, 2004. 209 p.

GREIG-SMITH, M. A. P. Quantitative plant ecology. 2. ed. London: Buther Worths, 1964. $256 \mathrm{p}$.

KAGEYAMA, P. Y.; CUNHA, G. C.; BARRETO, K. D.; GANDARA, F. B.; CAMARGO, F. R. A.; SEBBENN, A. M. Diversidade e autocorrelação genética espacial em populações de Ocotea odorífera (Lauraceae). Scientia Florestalis, Piracicaba, n. 64, p. 108-119, 2003.

KERSHAW, K. A. Quantitative and Dynamic plant ecology. 2. ed. New York: American Elsevier, 1973. 308 p.

KREBS, C. J. Ecological methodology. 2. ed. Menlo Park: Benjamim/Cummings, 1999. 620 p.

LEGENDRE, P.; FORTIN, M. J. Spatial pattern and ecological analysis. Vegetatio, London, v. 80, n. 2, p.107-138, 1989.

LUDWIG, J. A.; REYNOLDS, J. F. Statistical ecology: a primer on methods and computing. New York: John Wiley \& Sons, 1988. 368 p.

MIRANDA, I. S. Estrutura e fenologia de uma comunidade arbórea da savana amazônica de Alter-do-Chão, Pará. 1992. 129 f. Dissertação (Mestrado em Ciências Biológicas - Ecologia) - Fundação Universidade da Amazônia, Manaus, 1992.

MUELLER-DOMBOIS, D.; ELLENBERG, H. Aims and methods of vegetation ecology. New York: John Wiley \& Sons, 1974. 547 p.

OLIVEIRA, A. N. Variação genética entre e dentro de procedências de baru (Dipteryx alata Vog.). 1998. 80 f. Dissertação (Mestrado em Engenharia Florestal) - Universidade Federal de Lavras, Lavras, 1998.

O’SULLIVAN, D.; UNWIN, D. J. Geographic information analysis. 2. ed. New Jersey: John Wiley\& Sons, 2010. 432 p. 
PERRY, J. N.; DIXON, P. M. A new method to measure spatial association for ecological count data. Ecoscience, Philadelphia, v. 9, n. 2, p. 133-141, 2002.

RESENDE, M. D. V. SELEGEN- REML/BLUP: sistema estatístico e seleção genética computadorizada via modelos lineares mistos. Colombo: Embrapa Florestas, 2007. 561 p.

ROCHA, M. G. B.; ROCHA, D.; CLEMENTE, V. M. Teste de procedências e progênies de baru (Dipteryx alata Vog.). In: ROCHA, M. G. B. Melhoramento de espécies arbóreas nativas. Belo Horizonte: DDFS/Instituto Estadual de Florestas, 2002. p. 29-40.

ROSSETO, V.; ARAÚJO, J. S.; SFAIR, J. C.; LATINI, A. O. Avaliação da distribuição espacial de espécies arbóreas em um fragmento de Cerrado do município de Itirapina, SP. 2005.2 Disponível em: <http://www2.ib.unicamp.br/profs/fsantos/ecocampo/ne211/2005/R2-b.pdf>. Acesso em: 15 jul. 2016.

SANO, S. M.; RIBEIRO, J. F.; BRITO, M. A. Baru: biologia e uso. Planaltina: Embrapa Cerrados, 2004. 52 p. (Embrapa Cerrados. Documentos, 116).

SEBBENN, A. M.; KAGEYAMA, P. Y.; VENCOVSKY, R. Variabilidade genética, sistema reprodutivo e estrutura genética especial em Genipa americana L. através de marcadores isoenzimáticos. Scientia Forestalis, Piracicaba, v. 53, p.15-30, 1998.

SILVA, A. M., CANUTO, D. S. O., AGUIAR, A. V., MORAES, M. L. T. Autocorrelação espacial em uma população natural de piqui (Caryocar brasiliense Camb.) em área degradada. In: ENCONTRO DE GEÓGRAFOS DA AMÉRICA LATINA, 10, 2005, São Paulo. Anais... São Paulo: Universidade de São Paulo, 2005. p. 14233-14243,

SILVA, J. A.; SILVA, D. B.; JUNQUEIRA, N. T. V.; ANDRADE, L. R. M. Coleta de sementes, produção de mudas e plantio de espécies frutíferas nativas dos cerrados: informações exploratórias. Planaltina: Embrapa-CPAC, 1997. 23 p. (Embrapa-CPAC. Documentos, 44).

SIQUEIRA, A. C. M. F.; NOGUEIRA, J. C. B. Essências brasileiras e sua conservação genética no Instituto Florestal de São Paulo. In: CONGRESSO NACIONAL SOBRE ESSÊNCIAS NATIVAS, 2, 1992, São Paulo. Anais... [S. 1.: s. n.], 1992. p. 1187-1192.

SOUZA, L. M. F. I.; KAGEYAMA, P. Y.; SEBBENN, A. M. Estrutura genética em populações fragmentadas de Chorisia speciosa St. Hil (Bombacaceae). Scientia Forestalis, Piracicaba, v. 65, p.70-79, 2004.

THOMAS, C. D.; KUNIN, W. E. The spatial structure of populations. Journal of Animal Ecology, Chichester, v. 68, n. 4, p.647-657, 1999.

WALler, L. A.; GOTWAY, C. A. Applied spatial statistics for public health data. Hoboken: John Wiley\& Sons, 2004. 520 p.

ZARUMA, D. U. G.; CANUTO, D. S. O.; PUPIN, S.; CAMBUIM, J.; SILVA, A. M.; MORI, E. S.; SEBBENN, A. M.; MORAES, M. L. T. Variabilidade genética em procedências de Dipteryx alata Vogel para fins de conservação genética e produção de sementes. Scientia Forestalis, Piracicaba, v. 43, n. 107, p.609-615, 2015. 